\title{
Strong-field optoelectronics in solids
}

\author{
G. Vampa ${ }^{1,2 \star}$, T. J. Hammond ${ }^{1}$, M. Taucer ${ }^{1}$ Xiaoyan Ding', X. Ropagnol ${ }^{3}$, T. Ozaki ${ }^{3}$, S. Delprat $\oplus^{3}$, \\ M. Chaker ${ }^{3}$, N. Thiré ${ }^{3}$, B. E. Schmidt ${ }^{4}$, F. Légaré ${ }^{3}{ }^{3}$, D. D. Klug ${ }^{5}$, A. Yu. Naumov ${ }^{5}{ }^{5}$, D. M. Villeneuve ${ }^{5}$, \\ A. Staudte ${ }^{5}$ and P. B. Corkum ${ }^{1,5}$
}

\begin{abstract}
Perturbative optical nonlinearities induced by static electric fields ${ }^{1}$ have proven useful in visualizing dynamical function in systems including operating circuits ${ }^{2,3}$, electric and magnetic domain walls ${ }^{4}$, and biological matter ${ }^{5}$, and in controlling light for applications in silicon photonics ${ }^{6}$. Here, we extend field-induced second-harmonic generation to the non-perturbative regime. We demonstrate that static or transient fields up to terahertz ( $\mathrm{THz}$ ) frequencies applied to silicon and ZnO crystals generate even-order high harmonics. Images of the even harmonics confirm that static fields delivered with conventional electronics control the spatial properties of the high-harmonic emission. Extending our methodology to higher-harmonic photon energies ${ }^{7,8}$ paves the way for realizing active optics in the extreme ultraviolet and will allow imaging of operating electronic circuits ${ }^{9}$, of Si-photonic devices ${ }^{10}$ and of other functional materials $^{11,12}$, with higher spatio-temporal resolution than perturbative methods. For THz spectroscopy, our method has the bandwidth to allow measurement of attosecond transients imprinted on THz waveforms.
\end{abstract}

When intense light irradiates a crystal that lacks inversion symmetry, the induced dipole is different for one half-cycle of the driving laser field than for the following half-cycle, resulting in the emission of radiation at the second-harmonic frequency ${ }^{13}$. This asymmetric response can be mimicked in centrosymmetric crystals that are biased by a static electric field ${ }^{1}$ or a current ${ }^{2}$. The process can be interpreted as a third-order perturbative expansion of the nonlinear polarization at the second-harmonic frequency ${ }^{14}$, whereby one zero-frequency photon $\left(E_{\text {d.c. }}\right)$ adds to two photons of the fundamental laser frequency $\left(E_{\omega}\right)$ :

$$
P_{2 \omega} \propto \chi^{(3)} E_{\text {d.c. }} E_{\omega}^{2}
$$

where $\chi^{(3)}$ is the third-order nonlinear optical susceptibility. Applications of this effect for high-speed wide-field imaging of electric fields in devices ${ }^{2,3,15,16}$ and materials ${ }^{4,5}$ are limited to micrometre spatial resolution, given by the wavelength of the second harmonic (in the visible spectral range). Time-resolved electron microcopy can also map electromagnetic fields of similar magnitude ${ }^{17}$, potentially with attosecond temporal resolution ${ }^{18,19}$ and-owing to the short de Broglie wavelength of high-energy electrons-nanometre spatial resolution. Here we use d.c. and $\mathrm{THz}$ fields to generate even-order high harmonics from solids ${ }^{7,8,20-22}$, thereby extending field-induced optical nonlinearities to the non-perturbative regime. We demonstrate wide-field imaging of static fields with sensitivity as low as a few volts per micrometre from patterned $\mathrm{Si}$ and $\mathrm{ZnO}$ crystals. Given that harmonic wavelengths as short as $\sim 40 \mathrm{~nm}$ have been generated from other crystals ${ }^{7,8}$, our method bears potential for achieving nanometre spatial resolution. Although current electronics is smaller-scale still, solid harmonics could fulfil an important role because it is a wide-field method, is rather simple and is compatible with coherent diffractive imaging ${ }^{23}$, solving the lack of optical elements at extreme ultraviolet wavelengths. Turning measurement into control, we show that standard electronics controls the spatial properties of the high-harmonic beam, and can therefore be used to make reconfigurable optical elements for vacuum-ultraviolet radiation.

We fabricate Au electrodes on two different materials: a 40-nm (001)-oriented Si single crystal and a 500-nm (0001)-oriented $\mathrm{ZnO}$ single crystal, both on sapphire substrates (see Methods for details). Infrared laser pulses with a centre wavelength of $2 \mu \mathrm{m}$ are focused to an intensity of $\sim 0.6 \mathrm{TW} \mathrm{cm} \mathrm{cm}^{-2}$ in $\mathrm{Si}$ and $\sim 4 \mathrm{TW} \mathrm{cm}^{-2}$ in $\mathrm{ZnO}$ in the region surrounding the electrodes. Because of the nonlinear interaction, high-order harmonics are generated extending up to $6 \mathrm{eV}$ (harmonic 10, at the ultraviolet transmission limit of our spectrometer): see Fig. 1. In Si, the linear laser polarization is set parallel to the $<110>$ direction, where the nonlinear current is accelerated along the projection of the $\mathrm{Si}-\mathrm{Si}$ bonds on the (001) plane. This orientation maximizes the high-harmonic yield (Supplementary Fig. 1). A characterization of the high-harmonic emission process in $\mathrm{Si}$ is reported in the Supplementary Information (Supplementary Fig. 2). No significant dependence on crystallographic alignment is found from (0001)-oriented $\mathrm{ZnO}$. High-harmonic emission from $\mathrm{ZnO}$ has been characterized elsewhere ${ }^{20,21}$. When the electrodes are unbiased, only odd-order harmonics are generated, reflecting the inversion symmetry of the Si and (0001)-ZnO (red lines show a residual evenharmonic signal, whose origin is explained Fig. 1 caption). When the electrodes are biased, the symmetry of electron-hole trajectories is broken, and even harmonics up to the eighth order (a wavelength of $250 \mathrm{~nm}$ ) are detected (blue lines). The odd harmonics are unperturbed by the d.c. field.

In Fig. 2 we show a series of images recorded with an even or an odd harmonic when a static field is applied to silicon. Whereas the image recorded in the fifth harmonic (Fig. 2a,d) contains the shadow of the Au electrodes because the Au blocks the odd harmonic, images recorded with the fourth harmonic (shown in Fig. 2b,c,e,f) reveal the distribution of the static electric field around the electrodes. We see similar images in higher harmonics as well (Supplementary Fig. 3). The emission area, which is confined to a region near only one of the electrodes, may coincide with the depletion layer of the Schottky contact formed by the $\mathrm{Au} / \mathrm{Si}$ stack $^{24}$, which extends only $<10 \mathrm{~nm}$ from the idealized

'Department of Physics, University of Ottawa, Ottawa, Ontario, Canada. ${ }^{2}$ Stanford PULSE Institute, SLAC National Accelerator Laboratory, Menlo Park, CA, USA. ${ }^{3}$ INRS-EMT, Varennes, Quebec, Canada. ${ }^{4} \mathrm{few}$-cycle Inc., Montreal, Quebec, Canada. ${ }^{5}$ National Research Council of Canada, Ottawa, Ontario, Canada. *e-mail: gvampa@stanford.edu 


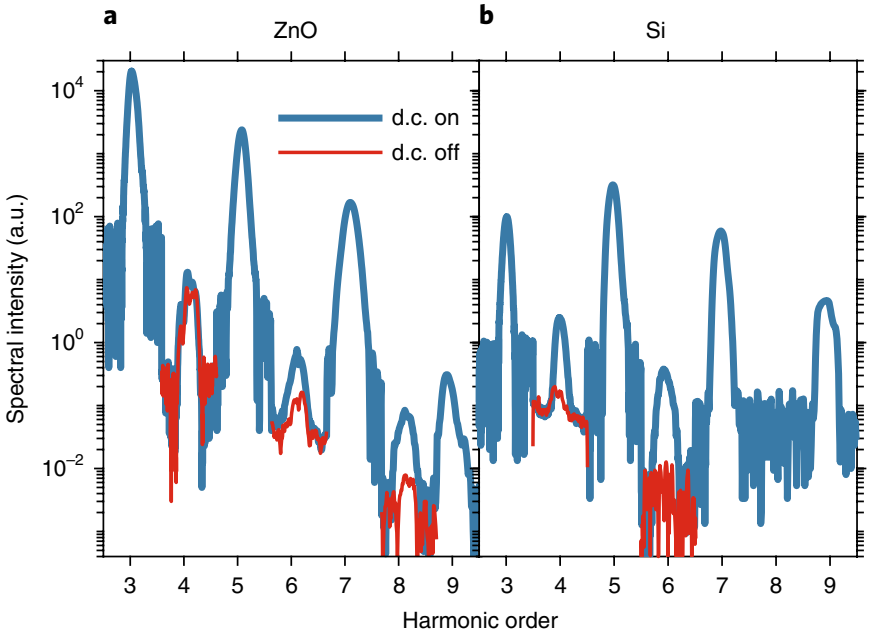

Fig. 1 | Breaking the symmetry with static fields. a,b, High-order even harmonics of a 2- $\mu \mathrm{m}$ driver are emitted from a $\mathrm{ZnO}$ (a) and a Si (b) crystal illuminated between two Au electrodes ( $40 \mu \mathrm{m}$ apart for $\mathrm{ZnO}$ and $100 \mu \mathrm{m}$ apart for $\mathrm{Si}$ ). One electrode is grounded and the other is biased to a $750-n s$ pulse (blue lines) at $316 \mathrm{~V}$ and $100 \mathrm{~V}$, respectively, for $\mathrm{ZnO}$ and $\mathrm{Si}$. The even-harmonic signal almost vanishes when the voltage is off (red lines). A weak residual even-harmonic signal from $\mathrm{ZnO}$ arises from contamination of the fundamental beam with a spurious second-harmonic field generated by the laser system. Each harmonic order is independently measured by a double-spectrometer $\left(\mathrm{a} \mathrm{CaF}_{2}\right.$ prism placed before a visible-ultraviolet spectrometer). The odd harmonics are unperturbed by the static field (not shown).

interface. Using a weak d.c. field with a component parallel to the driving field allows us to break the inversion symmetry of the oscillating dipole involved in the high-harmonic generation process, thereby generating even harmonics. Although it is possible to perturb the dipole with a perpendicular field ${ }^{25}$, stronger field strength is required to yield effects comparable to parallel polarization. Consequently, rotating the polarization of the driver from horizontal (Fig. 2b,d) to vertical (Fig. 2c,f) reveals the quadrature components of the field from which its vector can be obtained. We see that, for our prototypical device, the static field is polarized perpendicular to the edge of the Au electrodes, in the plane of the surface. The magnitude of the d.c. field is encoded in the relative brightness of the even and odd harmonics. The even harmonics have brightness $3 \%$ of that for the odd harmonics. Generating comparatively bright even harmonics by adding a known amount of second harmonic to the fundamental driver ${ }^{21,26}$ (see Supplementary Information), we can estimate a d.c. field strength of $\sim 13 \mathrm{~V} \mathrm{\mu m}^{-1}$. This sensitivity is comparable to other techniques ${ }^{15-17}$. Fields of this magnitude are present in modern electronic devices. The sign of the d.c. field can, in principle, be measured with the combined effect of d.c. and second-harmonic fields. The strongly anisotropic near-field emission of the fourth harmonic translates into markedly different propagation of the even harmonics compared with the odd harmonics. Therefore, we can control the spatial properties of a high-harmonic beam with electronic methods. Carefully designed electrodes will act as active optical elements, such as lenses and beam splitters, akin to electrostatic lenses for electron detection.

The even-harmonic signal increases quadratically with the applied voltage up to $300 \mathrm{~V}$ (Fig. 3a,b), which is consistent with both the perturbative (equation (1)) and the non-perturbative model (see Methods). However, despite the high order of the harmonics, the even-harmonic intensity increases linearly with the driving laser intensity (Fig. 3c), confirming that the even harmonics are non-perturbative with respect to the fundamental field. In fact, a perturbative $n$th harmonic requires $n$ photons of the fundamental and one d.c. photon, thereby yielding an intensity scaling of the harmonic proportional to the $n$th power of the intensity of the fundamental.

It is now a small step to dynamical images of time-dependent electric fields. We create a movie of a time-dependent electric field generated by applying a voltage pulse to electrodes and delaying the pulsed signal with respect to the laser pulse. Supplementary Video 1 shows how the field builds up on the electrodes. Here, the pulsed field is delayed in steps of $4 \mathrm{~ns}$ with respect to the laser pulse. The degree of fidelity of the high-harmonic measurement can be appreciated in Fig. 4a where we show the spectrally integrated signal from the sixth harmonic from $\mathrm{ZnO}$ as a function of delay with respect to the applied electrical pulse. Because the electric field is sensed only during the sub-laser cycle life of the electron-hole pair, the method will resolve electric fields from d.c. to $\mathrm{PHz}$ frequencies $^{27}$, as shown below.

In Fig. $4 \mathrm{~b}$ we demonstrate the potential for measuring highspeed electric field transients by recording the sixth harmonic (red line) generated when the $2-\mu \mathrm{m}$ fundamental beam overlaps a $\mathrm{THz}$ pulse (blue line) in a $\mathrm{ZnO}$ crystal (see Methods for details about the experimental set-up). Our measurements show that high-harmonic generation will be an important new probe of $\mathrm{THz}$ fields. High harmonics have advantages with respect to other $\mathrm{THz}$ methods, such as electro-optic sampling ${ }^{28}$, because phase matching is relaxed owing to the very small penetration depth of highharmonic radiation. In fact, in Fig. $4 \mathrm{~b}$ the electric field of the $\mathrm{THz}$ pulse measured with electro-optical sampling is also shown. For this measurement, we used a 2-mm-thick ZnTe electro-optic crystal (blue line). The retrieved pulse is longer than the pulse that we measure using high harmonics, probably as a result of improper phase matching in the thick ZnTe crystal. With appropriate temporal gating on the electron-hole excursion, high-harmonic sampling of $\mathrm{THz}$ waveforms will allow attosecond dynamics imprinted on a $\mathrm{THz}$ probe of materials to be retrieved, without using attosecond extreme-ultraviolet pulses.

Looking to the future, combining high-harmonic THz sampling with high harmonics perturbed by biased electrodes will enable us to provide interference of even harmonics from the $\mathrm{THz}$ pulse with those generated by the static field. Knowing the strength of the applied bias voltage, the peak field strength of a $\mathrm{THz}$ pulse can be directly evaluated. Compared with other coherent detection schemes of $\mathrm{THz}$ radiation ${ }^{29}$, which require strong-field ionization of gas atoms, solid-based high harmonics lowers the required infrared intensity by a factor of $\sim 100$.

In conclusion, high-harmonic generation in $\mathrm{ZnO}$ and $\mathrm{Si}$ - the most technologically relevant material-is an exquisitely sensitive electron-hole quantum sensor of the electric fields permeating materials. By localizing the emission very close to the electrodes, we have demonstrated basic control over the beam profile of the even harmonics. In the future, active optics for coherent vacuum-ultraviolet radiation - such as tunable lenses, beam splitters and mirrorscould potentially be realized by suitably designing the electrodes to achieve tailored customizable field distributions. These optics are available only at optical frequencies.

Dynamically imaging the even harmonics will enable us to record movies of electric fields in operating semiconducting and Si-photonic devices and near nanostructures with high temporal resolution and sensitivity of a few volts per micrometre, with the potential of high spatial resolution according to the short highharmonic wavelength. Chemically specific imaging can also be achieved, as suggested by a recent experiment that found a dependence of the high-harmonic intensity on doping the Si surface with Ga ions ${ }^{30}$. 


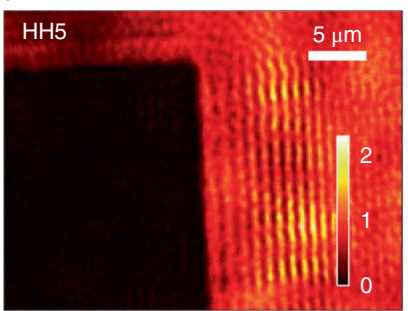

d

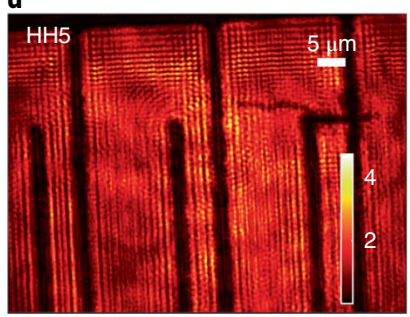

b

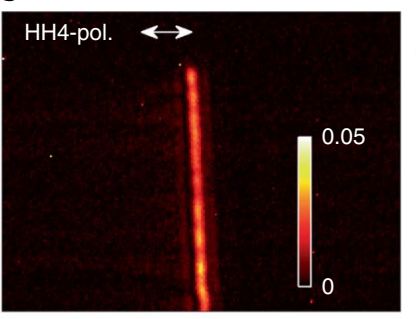

e

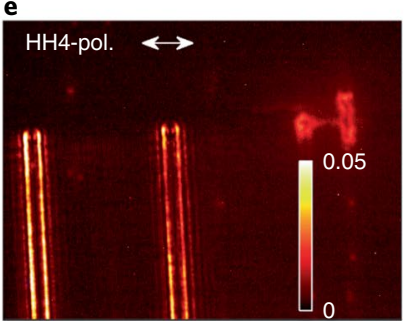

c

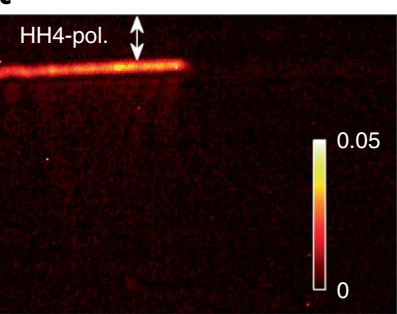

f

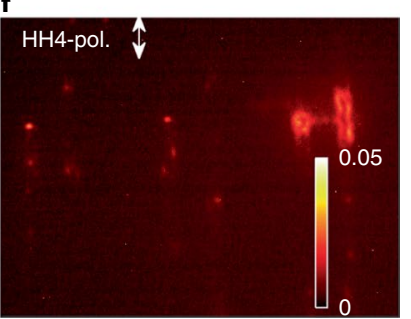

Fig. 2 I Imaging of fields in Si devices. a-f, Images of the fifth (a,d) and fourth harmonics (b,c,e,f) for two different designs of electrode: a single electrode ( $\mathbf{a}-\mathbf{c})$ and interdigitated electrodes ( $\mathbf{d}-\mathbf{f})$. In the latter case, only one row is biased. The fifth harmonic returns structural information: the electrodes (black) block high-harmonic emission from the underlying Si film. The fourth harmonic images the static electric field and its polarization. When the laser is horizontally (b,e) (vertically, c,f) polarized, only the field along the vertical (horizontal) sides of the electrodes is imaged. The linear colour scale encodes high-harmonic power, in arbitary units. The power in $\mathbf{b}, \mathbf{c}$ is relative to that of $\mathbf{a}$, and the power in $\mathbf{e}, \mathbf{f}$ is relative to that of $\mathbf{d}$. $\mathrm{HH}$, high harmonic; pol., polarized.

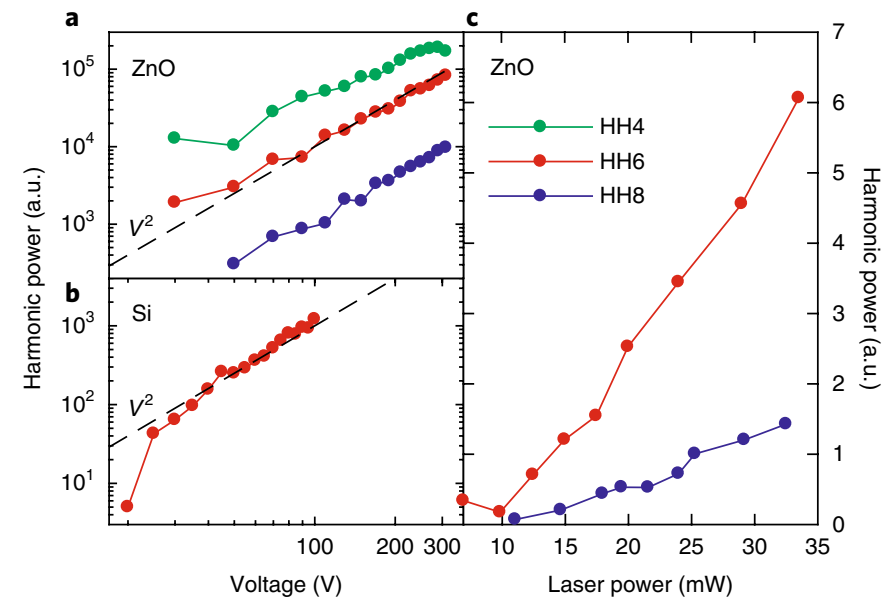

Fig. 3 | Extending even-harmonic generation to the non-perturbative regime. a,b, Intensity of even harmonics as a function of applied voltage $(\mathbf{a}, \mathrm{ZnO} ; \mathbf{b}, \mathrm{Si})$; $\mathbf{c}$, intensity as a function of fundamental laser intensity $(\mathrm{ZnO})$. All even harmonics scale quadratically with the applied voltage $(\mathbf{a}, \mathbf{b})$, in agreement with the non-perturbative model. The black dashed line is a guide to the eye for a quadratic scaling with voltage. In $\mathbf{c}$, the even harmonics scale linearly with the laser power. The linear scaling deviates from the typical perturbative power scaling $l^{n}$, where $n$ is the harmonic order.

\section{Methods}

Methods, including statements of data availability and any associated accession codes and references, are available at https://doi. org/10.1038/s41566-018-0193-5.

Received: 13 December 2017; Accepted: 17 May 2018; Published online: 18 June 2018

\section{References}

1. Terhune, R. W., Maker, P. D. \& Savage, C. M. Optical harmonic generation in calcite. Phys. Rev. Lett. 8, 404-406 (1962).

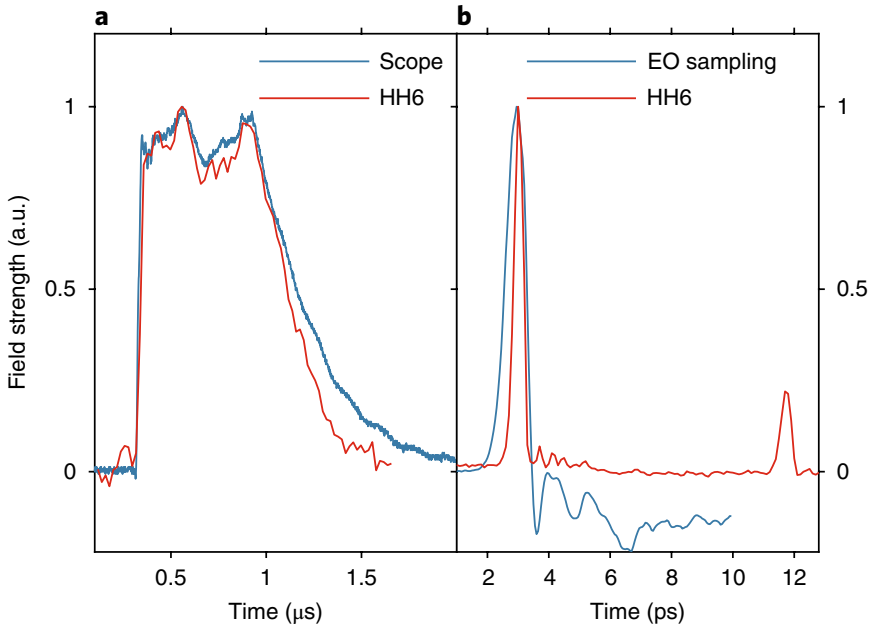

Fig. 4 | Sampling of time-dependent fields. a,b, The sixth harmonic (red line) from the $\mathrm{ZnO}$ crystal samples a pulsed static electric field ( $\mathbf{a}$, blue line, measured with an oscilloscope) and a half-cycle $\mathrm{THz}$ pulse (b, blue line, measured with electro-optic (EO) sampling in a 2-mm-thick ZnTe crystal with $800-\mathrm{nm}$ pulses acting as temporal gate). The square root of the harmonic signal follows the electric field (the harmonic signal scales quadratically with the applied perturbing field strength; see Fig. 3a). The peak $\mathrm{THz}$ pulse is estimated at $27 \mathrm{kV} \mathrm{cm}^{-1}$ with electro-optic sampling. The echo pulse at $\sim 12 \mathrm{ps}$ in $\mathbf{b}$ is consistent with a THz pulse that performs a round trip in the sapphire substrate, after being reflected from the output and entrance surfaces.

2. Ruzicka, B. A. et al. Second-harmonic generation induced by electric currents in GaAs. Phys. Rev. Lett. 108, 077403 (2012).

3. Manaka, T., Lim, E., Tamura, R. \& Iwamoto, M. Direct imaging of carrier motion in organic transistors by optical second-harmonic generation. Nat. Photon. 1, 581-584 (2007)

4. Fiebig, M., Lottermoser, T., Fröhlich, D., Goltsev, A. V. \& Pisarev, R. V. Observation of coupled magnetic and electric domains. Nature 419, 818-820 (2002). 
5. Peterka, D. S., Takahashi, H. \& Yuste, R. Imaging voltage in neurons. Neuron 69, 9-21 (2011).

6. Timurdogan, E. et al. Electric field-induced second-order nonlinear optical effects in silicon waveguides. Nat. Photon. 11, 200-206 (2017).

7. Luu, T. T. et al. Extreme ultraviolet high-harmonic spectroscopy of solids. Nature 521, 498-502 (2015).

8. You, Y. S., Reis, D. A. \& Ghimire, S. Anisotropic high-harmonic generation in bulk crystals. Nat. Phys. 13, 345-349 (2016).

9. Holler, M. et al. High-resolution non-destructive three-dimensional imaging of integrated circuits. Nature 543, 402-406 (2017).

10. Englund, D. et al. Controlling cavity reflectivity with a single quantum dot. Nature 450, 857-861 (2007).

11. Raja, A. et al. Coulomb engineering of the bandgap and excitons in two-dimensional materials. Nat. Commun. 8, 15251 (2017).

12. Tetienne, J.-P. et al. Quantum imaging of current flow in graphene. Sci. Adv. 3, e1602429 (2017)

13. Boyd, R. W. Nonlinear Optics (Academic Press, London, 2003).

14. Bloembergen, N. \& Pershan, P. S. Light waves at the boundary of nonlinear media. Phys. Rev. 128, 606-622 (1962).

15. Mourou, G. A. \& Meyer, K. E. Subpicosecond electro-optic sampling using coplanar strip transmission lines. Appl. Phys. Lett. 45, 492-494 (1984).

16. Valdmanis, J. A. $1 \mathrm{THz}$ bandwidth prober for high-speed devices and integrated circuits. Electron. Lett. 23, 1308-1310 (1987).

17. Ryabov, A. \& Baum, P. Electron microscopy of electromagnetic waveforms. Science 353, 374-377 (2016).

18. Feist, A. et al. Quantum coherent optical phase modulation in an ultrafast transmission electron microscope. Nature 521, 200-203 (2015).

19. Hassan, M. Th. et al. High-temporal-resolution electron microscopy for imaging ultrafast electron dynamics. Nat. Photon. 11, 425-430 (2017).

20. Ghimire, S. et al. Observation of high-order harmonic generation in a bulk crystal. Nat. Phys. 7, 138-141 (2011).

21. Vampa, G. et al. Linking high harmonics from gases and solids. Nature 522, 462-464 (2015).

22. Schubert, O. et al. Sub-cycle control of terahertz high-harmonic generation by dynamical Bloch oscillations. Nat. Photon. 8, 119-123 (2014).

23. Chapman, H. N. et al. Femtosecond diffractive imaging with a soft-X-ray free-electron laser. Nat. Phys. 2, 839-843 (2006).

24. Tung, R. T. Recent advances in Schottky barrier concepts. Mater. Sci. Eng. E 35, 1-138 (2001)

25. Niikura, H., Dudovich, N., Villeneuve, D. M. \& Corkum, P. B. Mapping molecular orbital symmetry on high-order harmonic generation spectrum using two-color laser fields. Phys. Rev. Lett. 105, 053003 (2010).
26. Dudovich, N. et al. Measuring and controlling the birth of attosecond XUV pulses. Nat. Phys. 2, 781-786 (2006).

27. Kim, K. K. et al. Petahertz optical oscilloscope. Nat. Photon. 7, 958-962 (2013).

28. Wilke, I. \& Sengupta, S. in Terahertz Spectroscopy: Principles and Applications (ed. Dexheimer, S. L.) 48-49 (CRC, Boca Raton, 2007).

29. Lu, X. \& Zhang, X.-C. Balanced terahertz wave air-biased-coherent-detection. Appl. Phys. Lett. 98, 151111 (2011).

30. Sivis, M. et al. Tailored semiconductors for high-harmonic optoelectronics. Science 357, 303-306 (2017)

\section{Acknowledgements}

We thank A. Laramée and C. A. Couture (Advanced Laser Light Source), D. Crane and B. Avery (NRC Canada) for technical support, and G. Lopinski (NRC Canada) for discussions and for lending electrical equipment. This material is based on work supported by the US Air Force Office of Scientific Research under award numbers FA9550-16-1-0109 and FA9550-15-1-0037; and by Canada's National Research Council (NRC), the Natural Sciences and Engineering Research Council (NSERC), Canada Foundation for Innovation (CFI) and the Ontario Research Fund (ORF).

\section{Author contributions}

G.V. and P.B.C. conceived the experiment. G.V., T.J.H., M.T., X.D. and N.T. performed the measurements with static fields. G.V. and X.R. performed the $\mathrm{THz}$ experiment. S.D. fabricated the electrodes. N.T., B.E.S. and A.S. maintained the laser sources. D.D.K. provided theoretical support. T.O., M.C., A.Yu.N., D.M.V., A.S., F.L. and P.B.C. supervised the experiments. All authors contributed to the manuscript.

\section{Competing interests}

The authors declare no competing interests.

\section{Additional information}

Supplementary information is available for this paper at https://doi.org/10.1038/ s41566-018-0193-5.

Reprints and permissions information is available at www.nature.com/reprints. Correspondence and requests for materials should be addressed to G.V.

Publisher's note: Springer Nature remains neutral with regard to jurisdictional claims in published maps and institutional affiliations. 


\section{Methods}

Fabrication of the electrodes. The electrodes are fabricated on the Si layer by a standard lift-off process: a 1.5- $\mu$ m-thick photoresist is spun and baked onto the sample, exposed on an EVG620 mask aligner under vacuum contact mode and developed into a tetramethylammonium-hydroxide-based solution. A 50-nm-thick Au layer (with a 5-nm-thick $\mathrm{Cr}$ adhesion layer) is then deposited on the patterned photoresist using electron-beam evaporation, and the sample is soaked in a solvent for few hours to complete the fabrication of the interdigitated electrodes.

Generation and detection of high harmonics. For the experiments presented in the main text, laser pulses $\sim 70 \mathrm{fs}$ long with a centre wavelength of $2.0 \mu \mathrm{m}$ are generated from a commercial optical parametric amplifier (LightConversion, TOPAS-Prime) pumped by $1.6-\mathrm{mJ}, 800-\mathrm{nm}$ laser pulses at $10 \mathrm{kHz}$ from a Coherent Legend Elite-CRYO System.

In the experiment presented in Fig. 4b, we generated even harmonics of a 2.1- $\mu \mathrm{m}$ femtosecond source with a synchronized $\mathrm{THz}$ pulse (centre frequency $\sim 0.15 \mathrm{THz}$ ). The $\mathrm{THz}$ pulse is generated by an interdigitated $\mathrm{ZnSe}$ large-aperture photoconductive antenna (LAPCA). The electrodes are made with $\mathrm{Cr}$ (adhesion layer) and $\mathrm{Au}$. The LAPCA is biased at $14 \mathrm{kV} \mathrm{cm}^{-1}$ with a $1-\mathrm{kV}, 20$-ns high-voltage pulse, synchronized with femtosecond $400-\mathrm{nm}$ pulses that photo-excite the LAPCA. The 400-nm pulses are obtained by frequency-doubling 800-nm pulses from a regenerative Ti:sapphire amplifier. Residual visible and infrared light is blocked by a black polyethylene sheet. The $\mathrm{THz}$ pulses are tightly focused on a 500 $\mathrm{nm} \mathrm{ZnO}$ film (grown over a 500 - $\mu \mathrm{m}$-thick sapphire substrate, $c$-axis cut), in which high harmonics are generated by $2.1-\mu \mathrm{m}$ pulses overlapped in space and time with the THz pulse. The THz pulse generates even harmonics up to the eighth order (the highest order that the spectrometer can detect).

Harmonics are detected with an Ocean Optics USB2000+ spectrometer up to a wavelength of $\sim 200 \mathrm{~nm}$.

Data availability. The data that support the plots within this paper and other findings of this study are available from the corresponding author upon reasonable request. 
In the format provided by the authors and unedited.

\section{Strong-field optoelectronics in solids}

\section{G. Vampa ${ }^{1,2 \star}$, T. J. Hammond', M. Taucer', Xiaoyan Ding ${ }^{1}$, X. Ropagnol ${ }^{3}$, T. Ozaki ${ }^{3}$, S. Delprat $\mathbb{B}^{3}$, M. Chaker ${ }^{3}$, N. Thiré ${ }^{3}$, B. E. Schmidt ${ }^{4}$, F. Légaré ${ }^{3}$, D. D. Klug ${ }^{5}$, A. Yu. Naumov ${ }^{5}$, D. M. Villeneuve ${ }^{5}$, A. Staudte ${ }^{5}$ and P. B. Corkum ${ }^{1,5}$}

\footnotetext{
'Department of Physics, University of Ottawa, Ottawa, Ontario, Canada. ${ }^{2}$ Stanford PULSE Institute, SLAC National Accelerator Laboratory, Menlo Park, CA, USA. ${ }^{3}$ NNRS-EMT, Varennes, Quebec, Canada. ${ }^{4} \mathrm{few}$-cycle Inc., Montreal, Quebec, Canada. ${ }^{5}$ National Research Council of Canada, Ottawa, Ontario, Canada. *e-mail: gvampa@stanford.edu
} 


\section{Strong-field optoelectronics in solids}

\section{Supplementary Information}

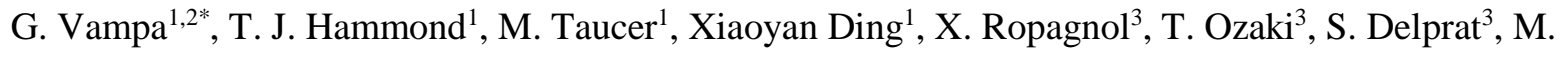

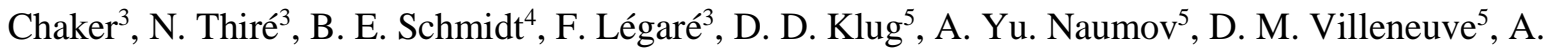
Staudte $^{5}$ and P. B. Corkum ${ }^{1,5}$

${ }^{1}$ Department of Physics, University of Ottawa, Ottawa, Ontario K1N 6N5, Canada

${ }^{2}$ Stanford PULSE Institute, SLAC National Accelerator Laboratory, Menlo Park, CA 94025, USA

${ }^{3}$ INRS-EMT, 1650 boulevard Lionel-Boulet, CP 1020, Varennes, Québec J3X 1S2, Canada

${ }^{4}$ few-cycle Inc, 2890 Beaurivage, Montreal, Québec H1L 5W5, Canada

${ }^{5}$ National Research Council of Canada, Ottawa, Ontario K1A OR6, Canada

*Corresponding author: gvampa@stanford.edu

Characterization of high-harmonic generation from silicon

In Supplementary Figure 1, we study the dependence of the high-harmonic power on the crystallographic orientation. We focus mid-infrared linearly polarized laser pulses of $100 \mathrm{fs}$ duration with a central wavelength of $3.5 \mu \mathrm{m}(0.35 \mathrm{eV}$ photon energy $)$ in a $500 \mathrm{~nm}$ thick Si single crystal, at a vacuum intensity of $\sim 0.6 \mathrm{TW} / \mathrm{cm}^{2}$. The film is epitaxially grown on a $500 \mu \mathrm{m}$ thick R-plane Sapphire substrate. The beam is incident on the silicon side, normal to the surface. The $\mathrm{Si}$ surface is (001). The [100] direction in the $\mathrm{Si}$ lattice is parallel to the projection of the c-axis of Sapphire on the surface ${ }^{31}$. Measurement of the birefringence of the substrate determines the orientation of this projection with respect to the laser polarization, which ultimately determines the orientation of the Si lattice with respect to the latter. The 4fold rotational symmetry of the Si crystal induces a clear symmetry of the emitted harmonics. Harmonic emission below the $13^{\text {th }}$ order is brightest for laser polarization in the $\langle 110\rangle$ direction, where the nonlinear current is accelerated along the projection of the Si-Si bonds on the (001) plane (see green arrow in inset of Supplementary Figure 1). The observed rotational dependence of high-harmonics can 
originate only from the microscopic generation process, because the linear optical properties of Si are

26 isotropic.

By perturbing the generation process with a weak second harmonic field added to the fundamental and observing the high-harmonic response ${ }^{21,26}$, we are able to calibrate the perturbing field strength. The addition of a second harmonic with an intensity of $9 \times 10^{-4}$ of the intensity of the strong mid-infrared driver - corresponding to a second-harmonic field strength of $\sim 30 \mathrm{~V} / \mu \mathrm{m}-$ generates even high-

32 harmonics with $\sim 20 \%$ the power of the odd harmonics, see Supplementary Figure 2. With the addition of 33 a DC field, even harmonics at the $\sim 3 \%$ level are generated. Therefore, given the quadratic scaling with 34 the perturbing field strength, we estimate the DC field strength to be $\sim 13 \mathrm{~V} / \mu \mathrm{m}$.

35 In the two-color experiment of Supplementary Figure 2, laser pulses of 55 fs duration with a central 36 wavelength of $3.7 \mu \mathrm{m}(0.33 \mathrm{eV}$ photon energy) are focused in a $40 \mathrm{~nm}$ Si single crystal at the same 37 intensity as in Fig. 1. The film is plasma etched from its initial thickness of $500 \mathrm{~nm}$. It is the same sample used for Fig. 1. Contrary to Supplementary Figure 1, the beam is incident on the Sapphire side, but to avoid birefringence the polarization is set parallel to the $\langle 100\rangle$ direction.

41 Besides being able to calibrate the DC field strength, we can use the second-harmonic perturbation to 42 characterize the high-harmonic emission process from Si. As seen in Supplementary Figure 2, the 43 intensity of each even high-harmonic modulates as we delay the second harmonic relative to the 44 fundamental. We find that, just as with gas harmonics ${ }^{26}$ and with $\mathrm{ZnO}^{21}$, the fundamental to second 45 harmonic phase difference that maximizes the intensity of the even high-harmonics varies slightly for 46 each order. This characteristic phase variation indicates that the mechanism responsible for high47 harmonic emission in silicon is the creation of an electron-hole pair near the minimum direct band gap (at $48 \quad 3.4 \mathrm{eV}$ in $\mathrm{Si}$ ) followed by acceleration along classical-like quantum trajectories until they re-collide and 
recombine, emitting a photon with energy determined by the bandgap at the crystal momentum of the pair

50 at the time of collision ${ }^{32}$.

$51 \quad$ Non-perturbative model for DC-field induced even-order high-harmonics

52 In a recollision model of high-order harmonic generation, the phase accumulated by the electron-hole pair 53 in the presence of a perturbing field $E_{2}$ is:

$$
\Phi=\Phi_{0}+\int_{t^{\prime}}^{t} \Delta v\left[k+A(t)-A\left(t^{\prime \prime}\right)\right]\left[A_{2}(t)-A_{2}\left(t^{\prime \prime}\right)\right] \mathrm{dt} "=\Phi_{0}+\sigma
$$

55 Where $\Phi_{0}$ is the high-harmonic dipole phase accumulated in the fundamental field only, $\Delta v(k)=$ $56 v_{c}(k)-v_{v}(k)$ is the difference in the conduction and valence band velocities (where $v=\nabla \epsilon(k), \epsilon(k)$ is

57 the band energy), $k$ is the crystal momentum, $A(t)$ is the vector potential of the driving laser field, and $A_{2}(t)$ is the vector potential of the perturbing field. The integral runs between the time of creation of the electron-hole pair by strong-field excitation $\left(t^{\prime}\right)$ and the time of recombination $(t)$. In the case of a static perturbing field, $A_{2}=-E_{D C} t$, so that:

$$
\sigma=E_{D C} \int_{t^{\prime}}^{t} \Delta v\left[k+A(t)-A\left(t^{\prime \prime}\right)\right]\left(t-t^{\prime \prime}\right) \mathrm{d} t^{\prime \prime}
$$

62 The intensity of the even harmonics ${ }^{26}$ is proportional to $|\sigma|^{2} \sim E_{D C}^{2}$, in agreement with Fig. 3a,b.

63 Moreover, we note that combining DC and second-harmonic fields will enable new methods for probing

64 the high-harmonic generation process. For example, the scaling of the even harmonic power with the DC-

65 field strength returns the integral of the electron-hole pair velocity over their lifetime. With the second harmonic alone, the same integrand is modulated by the vector potential of the second harmonic.

67 Combining this information one might be able to retrieve $t^{\prime}, t$ and $\Delta v$ with less assumptions than with the second-harmonic alone. 


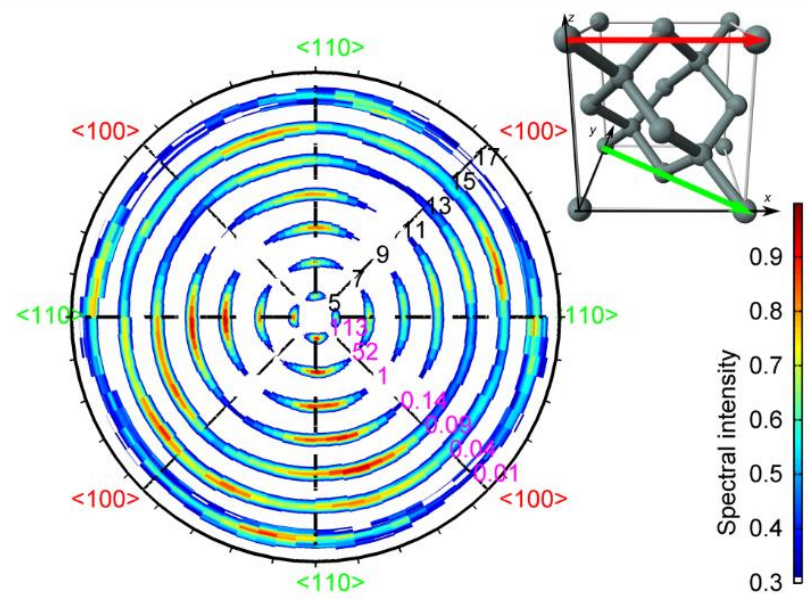

71 Supplementary Figure 1 | High-harmonic spectrum from Si. Polar plot of the emitted high-harmonic

72 spectral density generated from $\mathrm{Si}$. The azimuth corresponds to the incident polarization direction with

73 respect to the crystallographic axis, and the radius corresponds to the harmonic order (black labels,

74 extending from the $5^{\text {th }}$ to the $17^{\text {th }}$ order). The spectrum of each harmonic is plotted within $\pm 30 \%$ of each

75 odd harmonic order. Each harmonic order is separately normalized. The peak signal relative to the $9^{\text {th }}$

76 order is given by the magenta labels along the radial coordinate. The high-harmonic spectral density

77 modulates as the crystal is rotated with respect to the linear laser polarization. Maximum emission is

78 achieved for polarization along $\langle 110\rangle$, where the current is driven along the projection of the $\mathrm{Si}-\mathrm{Si}$ bond

79 on the (001) plane (green arrow in top-right inset). The red arrow in the top-right inset marks the direction

80 of the current for laser polarization along $\langle 100\rangle$. The laser center wavelength is $3.5 \mu \mathrm{m}$.

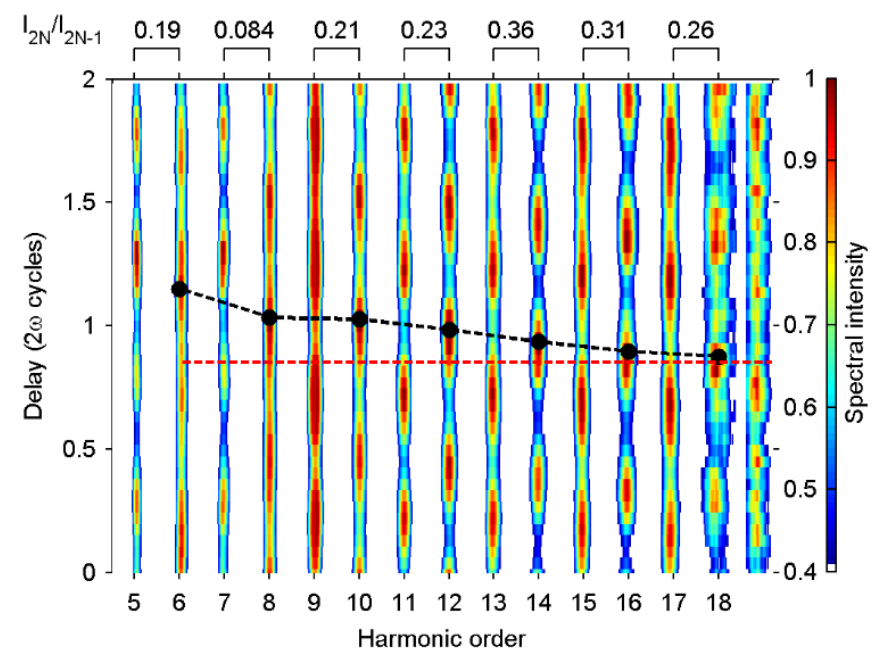


82 Supplementary Figure $2 \mid$ Adding a weak second harmonic frequency to the mid-infrared driver (center

83 wavelength of $3.5 \mu \mathrm{m}$ ) generates even-order harmonics from Si. The harmonic intensity modulates when

84 the delay between the driver and the second harmonic is varied. The phase of the modulation of the even

85 harmonics (black line) is markedly different between the orders, indicating a re-collision type emission

86 mechanism. Both the driver and its second harmonic are linearly polarized along the $\langle 110\rangle$ direction of

$87 \mathrm{Si}$, with the beam incident on the Si side to avoid birefringence induced by the Sapphire substrate. The

88 relative intensity of even and odd harmonic pairs is shown at the top. The signal in each order is

89 separately normalized.

90

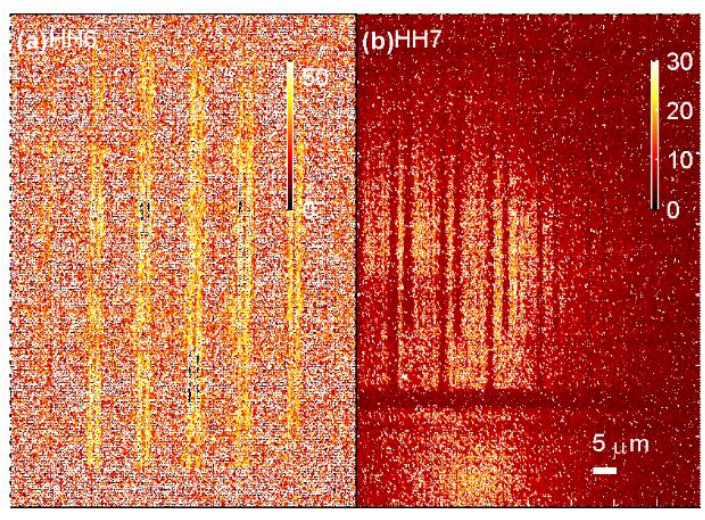

92 Supplementary Figure 3 | Imaging of electric fields is not limited to the $4^{\text {th }}$ harmonic. This figure shows

93 images of the $6^{\text {th }}$ (panel a) and $7^{\text {th }}$ (panel b) harmonics from a Si crystal when the electrodes are biased.

\section{References}

96 31. Chang, C. C. Silicon-on-sapphire epitaxy by vacuum sublimation: Leed-auger studies and electronic 97 properties of the films. J. Vac. Sci. Technol. 8, 500-511 (1971).

98 32. Vampa, G., et al. Theoretical analysis of high-harmonic generation in solids. Phys. Rev. Lett. 113, 073901 (2014). 